A-34-2020

\title{
RESPUESTA FISIOLÓGICA Y PRODUCTIVA DE POMELOS CV. 'STAR RUBY' A LA SUPRESIÓN DEL RIEGO DURANTE EL CRECIMIENTO DEL FRUTO
}

\author{
Berríos, Pablo (1). Gil, Ismael (1). Temnani, Abdelmalek (1). Pérez, David (1). Forcén, \\ Manuel (1). Zapata, Susana (1).Botía Ordaz, P. (3), Navarro Acosta, J.M. (3), Hernández \\ Ballester F.M. (3), Arques Pardo, E.M. (3). Pérez-Pastor, Alejandro (2).
}

1 Doctorando, Departamento de Ingeniería Agronómica, Universidad Politécnica de Cartagena, Cartagena, Región de Murcia, España. pablo.berrios@edu.upct.es

2 Catedrático, Departamento de Ingeniería Agronómica, Universidad Politécnica de Cartagena, Cartagena, Región de Murcia, España. alex.perez-pastor@upct.es

3 Equipo de Riego y Fisiología del Estrés, Instituto Murciano de Investigación y Desarrollo Agrario y Alimentario (IMIDA), La Alberca, Murcia), Región de Murcia, España.

\section{Resumen}

El aumento de la tensión por el uso de agua, la creciente escasez hídrica y la ocurrencia de eventos climáticos que destruyen infraestructura de riego en períodos críticos de los cultivos, hacen pertinente evaluar la sensibilidad de pomelo frente a diversos escenarios de disponibilidad hídrica, por ello se realizó un ensayo en una finca comercial de pomelos 'Star Ruby' en plena producción para evaluar el efecto de la supresión del riego durante el crecimiento del fruto sobre la producción, calidad y comportamiento post recolección. Los tratamientos aplicados fueron: (i) un control (CTL), regado para mantener los valores de potencial hídrico de tallo ( $\Psi$ tallo) a mediodía en torno a $-1 \mathrm{MPa}$; dos tratamientos de estrés hídrico, en los que se regaron como CTL, excepto (ii) IS II, durante los días 82 y 127 días desde máxima floración (DDMF), correspondientes a la fase II de crecimiento del fruto, y (iii) IS III, durante los días 180 y 262 DDMF, correspondientes a la fase III de crecimiento del fruto, en los que se suprimió el riego hasta alcanzar un umbral de $\Psi$ tallo en torno a -2,5 MPa. La integral de estrés hídrico obtenida para cada tratamiento fue de 26.8 y $13.6 \mathrm{MPa}$ día $^{-1}$ para IS II y III, respectivamente, no pudiéndose generar un similar nivel de estrés para ambos tratamientos. La supresión de riego permitió obtener un ahorro de agua de riego en torno al $20 \%$ sin afectar negativamente a la producción ni a la calidad, exceptuando que los frutos del IS III presentaron una concentración de sólidos disueltos mayor, en torno a un $7 \%$, y una tendencia de sus frutos a adquirir un color amarillo. El comportamiento post recolección fue similar entre tratamientos, aunque los frutos sometidos a supresión de riego durante fase II presentaron una ligera mayor firmeza a los 60 días después de la recolección.

\section{"Abstract"}

The increase of tension for the use water, water scarcity and the occurrence of climatic events that destroy irrigation infrastructure in critical periods of crops phenology, make it relevant to evaluate the sensitivity of grapefruit against various scenarios of water availability, so a trial was conducted in a commercial farm of grapefruit 'Star Ruby' in full production to assess the effect of irrigation suppression during the growth of the fruit on production, quality and post harvest behavior. The treatments applied were: (i) a control (CTL), irrigated to maintain midday stem water potential values $(\Psi$ stem) around $-1 \mathrm{MPa}$; two water stress treatments, in which they were irrigated as CTL, except (ii) IS II, during the days 82 and 127 days from maximum flowering (DFMF), corresponding to phase II of fruit growth, and (iii) IS III, 
during the days 180 and 262 DFMF, corresponding to phase III of fruit growth, in which irrigation was suppressed until reaching a threshold of $\Psi$ stem around $-2.5 \mathrm{MPa}$. The integral of hydric stress obtained for each treatment was 26.8 and $13.6 \mathrm{MPa}$ day-1 for IS II and III, respectively, not being able to generate a similar level of stress for both treatments. Irrigation supression allowed to obtain a saving of irrigation water around $20 \%$ without affecting negatively the production and quality, except that the fruits of IS III presented a higher concentration of dissolved solids, around $7 \%$, and a tendency of its fruits to acquire a yellow color. Post-harvest behavior was similar between treatments, although fruits subjected to irrigation suppression during phase II showed a slightly higher firmness 60 days after harvest.

\section{1- Introducción}

El cultivo de pomelo (Citrus paradisi Macf) ha ido adquiriendo relevancia a lo largo de los últimos años en la Región de Murcia, con un incremento de la superficie cultivada del $35.9 \%$ entre los años 2012 y 2018 ( 904 ha). Por otro lado, existen predicciones de un incremento de la escasez hídrica por efecto del cambio climático en diversas zonas productivas, provocando una creciente tensión por el agua entre la agricultura y otros sectores productivos. Asimismo, la ocurrencia de eventos climáticos como las DANAs por el acrónimo de "Depresión Aislada de Niveles Altos" que dan lugar a precipitaciones torrenciales y de díficil previsión (León, 2003) han causado diversas inundaciones, daños materiales y alertas en distintas áreas agrícolas. En concreto, durante el mes de septiembre de 2019 tuvo lugar este fenómeno climático en gran parte de la Región de Murcia, provocando rotura de importantes infraestructuras de riego de la región, impidiendo el suministro hídrico durante varias semanas a las zonas afectadas (Manuel Buitrago, 2019; P.B. / EFE, 2019) que coincidieron, en particular, con el período de crecimiento del fruto en cítricos. Por todo esto, y dado el escenario de reducida disponibilidad hídrica durante la temporada, se hace necesario evaluar la sensibilidad del cultivo frente a la supresión total del riego durante estados de desarrollo que no afecten la producción, calidad y comportamiento post recolección de la fruta.

\section{2- Objetivos}

Evaluar la respuesta agronómica y fisiológica de pomelo cv. Star Ruby frente a la supresión del riego durante las fases II y III de crecimiento del fruto, y su incidencia en la producción, calidad y comportamiento de la cosecha en post-recolección.

\section{3- Materiales y Métodos}

El estudio se llevó a cabo durante 2019 en una finca comercial en plena producción, con pomelos (Citrus paradisi Macf) cv. 'Star Ruby', injertados sobre Citrus macrophylla ubicada en Campotéjar (Murcia, España). La plantación fue establecida en 2013 a un marco de plantación de $6 \times 4 \mathrm{~m}$. Las principales técnicas culturales, excepto el riego, se realizaron bajo el criterio del agricultor. El sistema de riego consistió de un lateral por hilera de árboles y 4 emisores de $4.0 \mathrm{~L} \cdot \mathrm{h}^{-1}$ por árbol. La máxima floración tuvo lugar el 24 de abril de 2019.

Los tratamientos aplicados fueron: (i) un control (CTL), regado para mantener los valores de potencial hídrico de tallo ( $\Psi$ tallo) a mediodía en torno a $-1 \mathrm{MPa}$ (Conesa et al., 2018); dos tratamientos de estrés hídrico, en los que se regaron como CTL, excepto (ii) IS II, durante los días 82 y 127 días desde máxima floración (DDMF), correspondientes a la fase II de crecimiento del fruto, y (iii) IS III, durante los días 180 y 262 DDMF, correspondientes a la fase III de crecimiento del fruto, en los que se suprimió el riego hasta alcanzar un umbral de $\Psi$ tallo en torno a -2,2 MPa.

El contenido volumétrico de agua en el suelo $(\Theta v)$ se midió con sensores FDR (ECH20 10HS, METER Group Inc., EE.UU.) y el potencial matricial con sensores del potencial dieléctrico del agua en el suelo (MPS-6, Decagon Devices Inc., EE.UU.), a partir de un sensor 
por repetición para cada variable instalados a $0.4 \mathrm{~m}$ de profundidad y a $0.2 \mathrm{~m}$ de distancia del emisor ubicado bajo la canopia del árbol. Өv mínimo diario se relativizó respecto a la capacidad de campo (CC), que fue obtenida tras la estabilización de la humedad.

Utallo se midió a mediodía solar con una cámara de presión tipo Scholander modelo Pump-Up (PMS Insrument Company, EE.UU.) en tres hojas adultas por repetición y sin daños aparentes y sombreadas. Las hojas fueron cubiertas mediante una bolsa con revestimiento de aluminio $1.5 \mathrm{~h}$ antes de la medida. La integral de estrés hídrico se determinó a partir de la ecuación 1.

(Ec. 1)

$$
S_{\Psi}=\left|\sum_{i=0}^{i=i}\left(\Psi_{i, i+1}-\Psi_{C T L}\right) n\right|,
$$

Donde: $S_{\Psi}\left(\mathrm{MPa}\right.$ día $\left.^{-1}\right)$, integral de estrés hídrico; $\Psi_{i, i+1}(\mathrm{MPa})$, potencial hídrico de tallo para cada intervalo i, i+1; $\Psi_{\text {CTL }}(\mathrm{MPa})$, potencial hídrico de tallo correspondiente al tratamiento CTL; y $n$, número de días entre dos medidas consecutivas.

La evapotranspiración de referencia (ETo) y precipitaciones se obtuvieron a través de la estación Molina de Segura "Campotejar" perteneciente a la red del Sistema de Información Agrario de Murcia (SIAM, 2019).

El diámetro ecuatorial del fruto se midió con un calibre digital (Mitutoyo America Corporation, EE.UU.) en 75 frutos por repetición seleccionados al azar alrededor de la canopia.

La recolección de todos los frutos se realizó el día 10 de enero de 2020 (262 DDMF) a partir de un criterio comercial en todos los árboles testigo, en los que se determinó el rendimiento (Kg por árbol y t por ha), carga frutal (número de frutos por árbol) y peso medio del fruto. La eficiencia en el uso del agua de riego (EUAr, $\mathrm{Kg} \mathrm{m}^{-3}$ ) se determinó como la relación entre la producción por árbol y el volumen de agua de riego utilizado. Paralelamente se escogieron al azar 5 frutos por repetición, en los que se realizó el estudio físico y químico. La proporción de zumo, pulpa y piel en base a peso; y el zumo se evaluó según la metodología descrita por (Kimball, 1991). El color de la epidermis se determinó con un fotocolorímetro por reflexión triestímulo (Minolta CR-300) y se expresaron como valores de CIE $L^{*}, a^{*}$ y $b^{*}$ (Artés et al., 1997). Entre los parámetros químicos se incluyeron $\mathrm{pH}$, sólidos solubles (SS), acidez del zumo por neutralización con $\mathrm{NaOH} 0.1 \mathrm{~N}$ y el índice de madurez (IM) como la relación entre los sólidos solubles y la acidez (Artés et al., 1997).

Después de la recolección, se conservaron 50 frutos por repetición durante 55 días entre 8 y $10{ }^{\circ} \mathrm{C}$ de temperatura y entre 80 y $90 \%$ de humedad relativa, y posteriormente durante 7 días, en condiciones ambientales. La temperatura y humedad relativa diaria se registraron mediante un termohigrómetro digital modelo LOG210 (Dostmann Electronics, Alemania). Se registró cada 15 días la evolución del peso fresco y firmeza $\left(\mathrm{Kg} \mathrm{cm}^{-2}\right)$, con un penetrómetro modelo FT-20 (Wagner Instruments, Estados Unidos).

Se estableció un diseño experimental con 3 repeticiones por tratamiento distribuidas de forma aleatoria, con 3 árboles por repetición. Se realizó un análisis de varianza y cuando se detectaron diferencias entre tratamientos las medias se separaron mediante la prueba de comparaciones múltiples de Duncan a un $5 \%$ de significancia.

\section{4- Resultados y Discusión}

El volumen de agua de riego total utilizada para la temporada de estudio se redujo un 21.9 y $17.3 \%$ para IS II y III, respectivamente (Figura 1). La demanda climática en los dos ciclos de estrés hídrico fue muy diferente, de esta manera, durante IS II la evapotranspiración de referencia (ETo) osciló entre 5 y $7 \mathrm{~mm}$ por día, mientras que en IS III, estos valores fueron muy inferiores, oscilando entre 2 y $3 \mathrm{~mm}$ por día (Figura 1). Además, durante la semana 21 después de máxima floración y previo al inicio de IS III, ocurrieron precipitaciones acumuladas de $249.8 \mathrm{~mm}$. Por estos motivos, el tratamiento IS II indujo un mayor estrés hídrico en la planta 
que el correspondiente a IS III, de esta forma, la integral de estrés hídrico acumulada $\left(S_{\Psi}\right)$ durante el ciclo de cultivo correspondió a 26.8 y $13.6 \mathrm{MPa}^{\text {día }}{ }^{-1}$ para IS II y III, respectivamente.

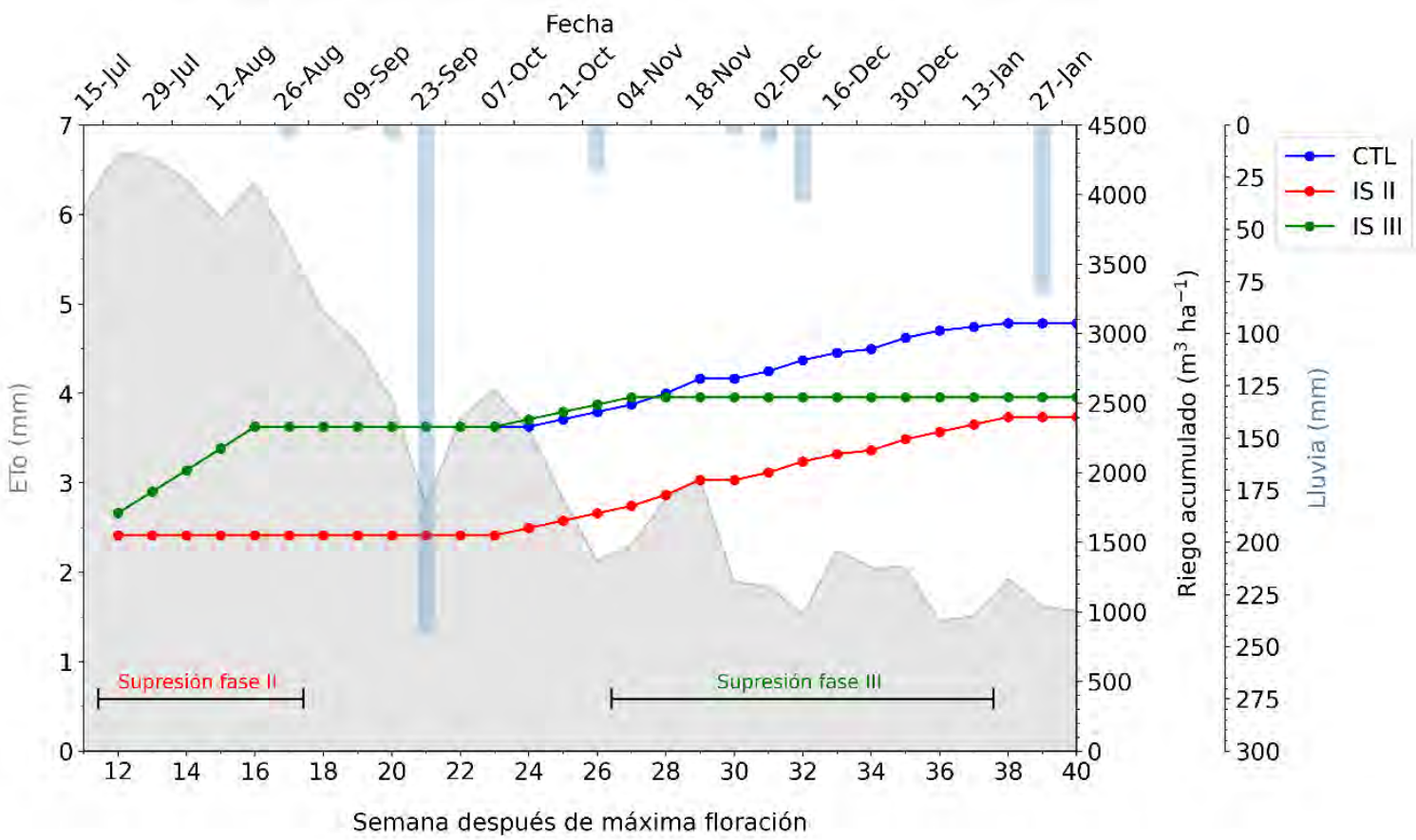

Figura 1. Parámetros climáticos durante los ciclos de supresión de riego y volumen de agua de riego acumulada.

No se detectaron diferencias en la carga frutal (número de frutos por árbol) entre tratamientos, aunque con cierta variabilidad en los valores promedios., siendo la media observada entre tratamientos de 832 frutos por árbol (Tabla 1).

El peso y diámetro ecuatorial del fruto no se vieron afectados por los ciclos de estrés hídrico y se obtuvieron valores medios entre tratamientos correspondientes a frutos de 338.2 $\mathrm{g}$ de peso con un diámetro ecuatorial de $93.71 \mathrm{~mm}$ (Tabla 1).

Dado que la carga frutal fue similar en las plantas evaluadas y no existió efecto de los tratamientos sobre las características físicas del fruto a recolección, no se detectaron diferencias significativas en el rendimiento. El tratamiento IS III presentó un rendimiento ligeramente superior pero a la vez una mayor carga frutal, aunque sin diferenciarse significativamente (Tabla 1).

Ambos tratamientos de supresión hídrica permitieron incrementar significativamente la eficiencia del uso del agua de riego (EUAr) respecto al control y no se detectaron diferencias entre ellos, a pesar que la mayor EUAr correspondió a IS II. Los ciclos de supresión permitieron incrementar la EUAr un $29.5 \%$ (Tabla 1).

Tabla 1. Componentes del rendimiento y eficiencia del uso del agua de riego (EUAr).

\begin{tabular}{|c|c|c|c|c|c|c|c|c|}
\hline \multirow{2}{*}{ Tratamiento } & \multicolumn{2}{|c|}{ Rendimiento } & \multirow{2}{*}{$\begin{array}{c}\begin{array}{c}\text { Peso } \\
\text { fruto } \\
g\end{array}\end{array}$} & \multirow{2}{*}{$\begin{array}{c}\text { Diámetro } \\
\text { ecuatorial } \\
\mathrm{mm}\end{array}$} & \multirow{2}{*}{\multicolumn{2}{|c|}{$\begin{array}{l}\text { Carga frutal } \\
\text { Frutos arbol }^{-1}\end{array}$}} & \multirow{2}{*}{\multicolumn{2}{|c|}{$\begin{array}{l}\text { EUAr } \\
\mathrm{Kg} \mathrm{m}^{-3}\end{array}$}} \\
\hline & $\mathrm{Kg}$ árbol-1 & $\mathrm{tha}^{-1}$ & & & & & & \\
\hline $\mathbf{C}$ & $274.11 \quad a$ & $114.2 \quad \mathrm{a}$ & 332,02 a & $92.80 \quad a$ & 826.8 & $a$ & 37.1 & $\mathrm{~b}$ \\
\hline & $281.10 \quad a$ & 117.1 & 356,99 a & $95.55 \quad$ a & 78 & a & 48 & a \\
\hline IS I & $288.66 \quad a$ & $120.2 \mathrm{a}$ & $325.61 \mathrm{a}$ & $92.78 \quad$ a & 886.8 & $a$ & 47.3 & a \\
\hline
\end{tabular}

Diferentes letras dentro de cada columna indican diferencias significativas según la prueba de Duncan $(p<0.05)$.

La diferencia de $\Psi$ tallo de IS II respecto al control fue significativamente menor durante la mayor parte del ciclo de estrés y se observó una máxima diferencia al alcanzar el umbral 
preestablecido de riego (en torno a $-2.2 \mathrm{MPa}$ ) correspondiente a $0.85 \mathrm{MPa}$ en la semana 18 después de máxima floración. Si bien las diferencias de $\Psi$ tallo entre IS III y CTL durante el ciclo de estrés fueron significativas, éstas fueron muy inferiores a las de IS II y no superaron $0.2 \mathrm{MPa}$ (Figura 2). El mínimo observado en IS III correspondió a -1 MPa durante la semana 33 después de máxima floración.

En ambos tratamientos, una vez que el potencial matricial del suelo fue significativamente menor que CTL, se observaron diferencias significativas en el $\Psi$ tallo, pero sólo se observó un incremento de la diferencia en el $\Psi$ tallo respecto al control en IS II una vez que el menor potencial matricial fue sostenido durante el ciclo; sin embargo, este efecto fue más amortiguado en IS III, tal y como ocurrió con los valores del contenido de agua en el suelo respecto a $\mathrm{CC}$, en ambos ciclos, con un valor mínimo similar en ambos ciclos (0.65), pero más continuado en el tiempo en IS II (Figura 2).
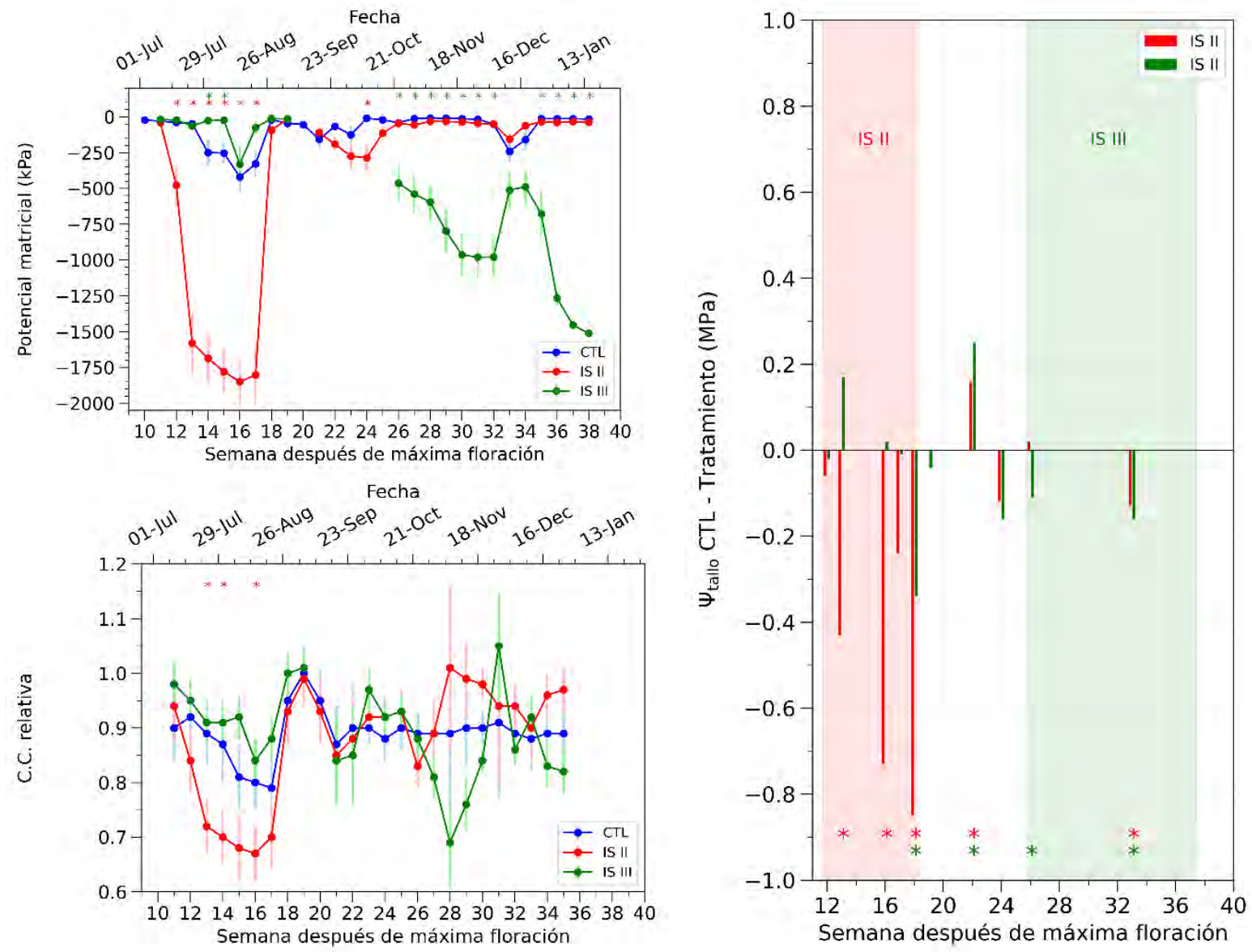

Figura 2. (A) Evolución del potencial matricial y (B) capacidad de campo relativa del suelo. (C) Diferencias observadas entre potencial de tallo de CTL y los tratamientos durante los ciclos de estrés hídrico. Medias \pm error estándar. * $y$ * indican diferencias respecto a CTL para IS II y III, respectivamente en una misma fecha según la prueba de Duncan $(p<0.05)$.

Respecto a la calidad del fruto, el porcentaje de zumo y piel no se vieron afectados por la supresión del riego, pero la proporción de pulpa fue mayor en la supresión durante la fase Il con respecto al control. El índice de madurez es adecuado para establecer un criterio sobre la madurez interna del fruto puesto que a medida que éste se acerca a la madurez, la acidez disminuye por el catabolismo del ácido citrico y los sólidos solubles totales se incrementan (Navarro et al., 2015; Pérez-Pérez et al., 2009). No se observó un retraso sobre la maduración por los tratamientos de supresión hídrica, ni sobre la acidez titulable ni los azúcares totales por separado. En árboles de pomelo sometidos a estrés hídrico, (Navarro et al., 2015) 
observaron que un estrés hídrico severo (100 MPa día-1 $)$ durante la fase II retrasa la madurez interna debido al incremento de la acidez y disminución de azúcares, a diferencia de un estrés en la fase III que adelantó la maduración por una mayor contenido de azúcares. Esta tendencia se observó en el presente estudio pero la madurez no se vió afectada porque también la acidez en IS III fue superior, aunque no siginificativa. Cuando la intensidad del estrés hídrico fue inferior a $70 \mathrm{MPa}_{\text {día }}{ }^{-1}$, los autores (Navarro et al., 2015) no observaron efectos sobre la maduración, por lo que es esperable que la severidad del estrés aplicado en ISII respecto al CTL no tenga un efecto sobre IM, dado que la menor intensidad de estrés alcanzada (Figura 3).

Respecto a la madurez exterior del fruto, evaluada como color de la epidermis, IS III presentó frutos de mayor luminosidad y más cercanos al color amarillo (Tabla 2).

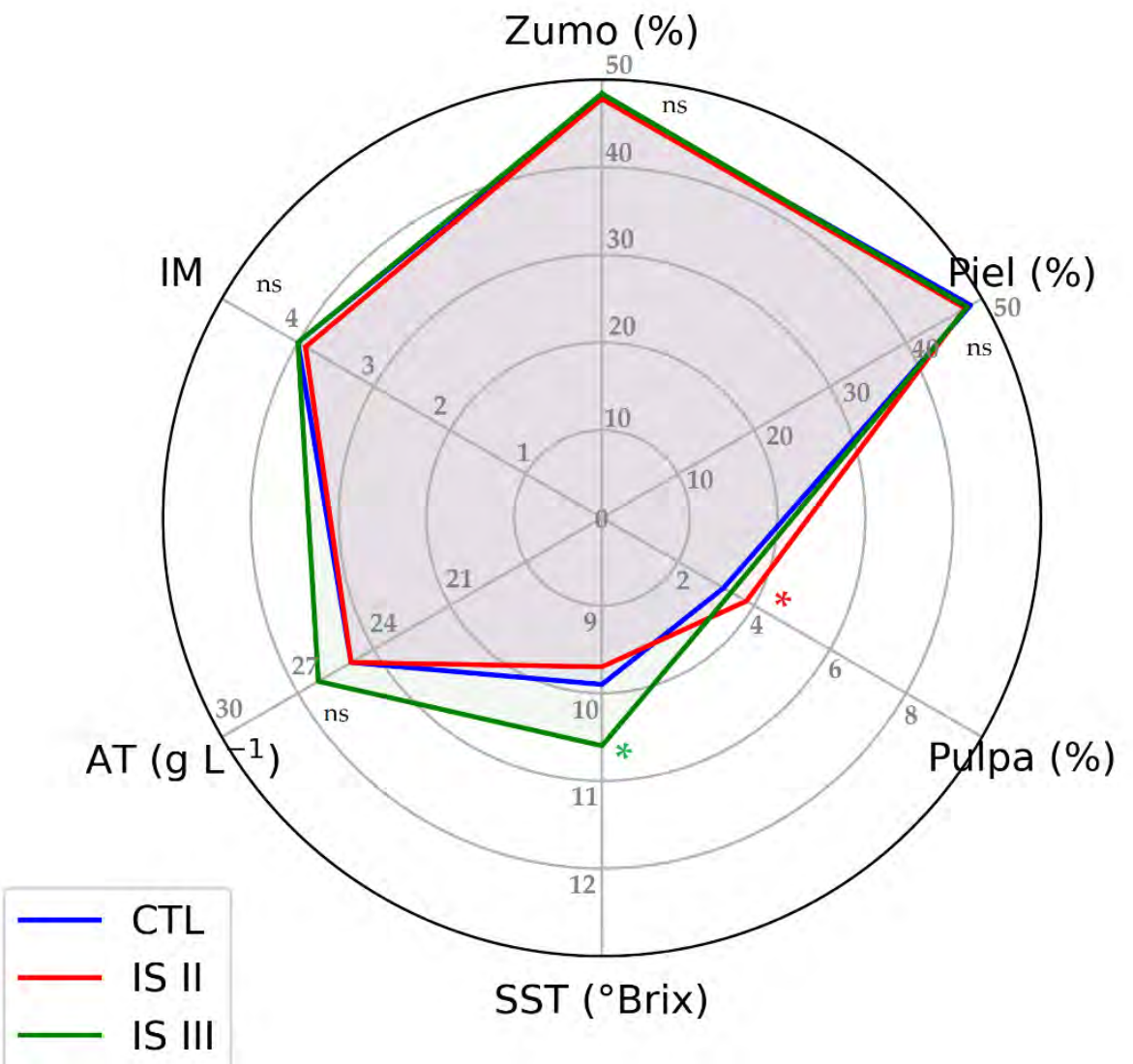

Figura 3. Componentes de la calidad de fruto durante recolección. SST: sólidos solubles totales; AT: acidez titulable e IM: índice de madurez. * $y$ * indican diferencias respecto a CTL para IS II y III, respectivamente en una misma fecha según la prueba de Duncan $(p<0.05)$. ns: no significativo.

Tabla 2. Color de la epidermis a recolección.

\begin{tabular}{c|ccccccc}
\hline \multirow{2}{*}{ Tratamiento } & \multicolumn{6}{|c}{ CIE } \\
& \multicolumn{6}{|c}{$\mathrm{L}$} & \multicolumn{2}{c}{$\mathrm{a}$} & \multicolumn{2}{c}{$\mathrm{b}$} \\
\hline CTL & 64.3 & $\mathrm{a}$ & 12.0 & $\mathrm{a}$ & 30.2 & $\mathrm{a}$ \\
IS II & 65.0 & $\mathrm{a}$ & 11.5 & $\mathrm{a}$ & 30.3 & $\mathrm{a}$ \\
IS III & 66.6 & $\mathrm{~b}$ & 11.4 & $\mathrm{a}$ & 31.7 & $\mathrm{~b}$ \\
\hline
\end{tabular}

CIE: espacio de color. Diferentes letras dentro de cada columna indican diferencias significativas según la prueba de Duncan $(p<0.05)$. 
No se observaron diferencias entre tratamientos en la deshidratación del fruto almacenado. El peso del fruto disminuye una media de $15 \%$ los primeros 15 días después de recolección para mantenerse relativamente constante hasta que se elimina el tratamiento de frío a los 54 días después de recolección dónde el peso se reduce alrededor de un $10 \%$ del final en frío. La firmeza ecuatorial del fruto se mantiene estable entre 30 y 40 días después de recolección y disminuye al terminar el proceso de frío, dónde el tratamiento IS II presentó una firmeza significativamente mayor al resto de los tratamientos (Figura 4).

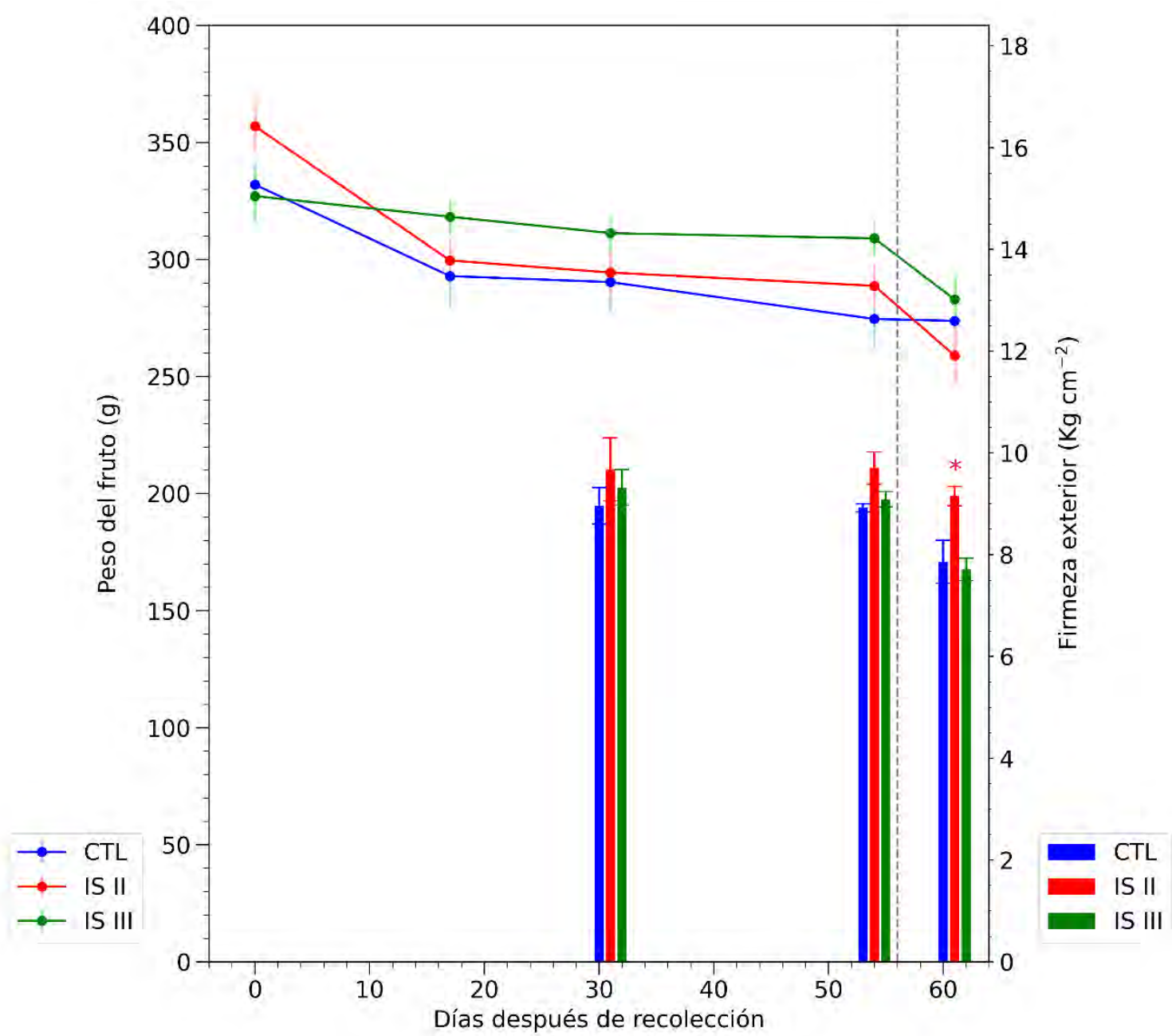

Figura 4. Evolución de la calidad post recolección del fruto. Las barras verticales y puntos indican el peso medio y la firmeza del fruto, respectivamente. La línea vertical punteada indica el fin del almacenaje en frío. * $y$ * indican diferencias respecto a CTL para IS II y III, respectivamente en una misma fecha y parámetro según la prueba de Duncan $(p<0.05)$.

\section{4- Conclusiones y Recomendaciones}

No se pudo generar el mismo nivel de estrés hídrico en ambos ciclos, debido a la variación en la demanda climática y las precipitaciones ocurridas en septiembre que retrasaron la aplicación del estrés hídrico en la fase III.

Los tratamientos de supresión permitieron ahorrar en torno a $20 \%$ el agua de riego utilizada durante la temporada.

La supresión del riego durante la fase II o III de crecimiento de fruto para las integrales de estrés obtenidas, no afectaron negativamente la producción y permitieron incrementar la eficiencia del uso del riego conjuntamente un 29.5\%. Estos resultados debieran ser comprobados el próximo ciclo de cultivo para evaluar un posible efecto sobre la carga frutal 
venidera. Con respecto a los indicadores, todos mostraron una elevada sensibilidad al estrés hídrico, si bien tanto el potencial hídrico de tallo como el matricial del agua en el suelo, fueron los que primero detectaron el inicio del estrés en IS II.

Los tratamientos no afectaron la fecha de recoleccíón ni el índice de madurez de la fruta, aunque se observó que los frutos sometidos a una supresión de riego durante fase III presentaron un $7 \%$ más de sólidos solubles totales y una tendencia hacia el color amarillo.

El comportamiento post recolección fue similar entre tratamientos, aunque los frutos sometidos a supresión de riego durante fase II presentaron una ligera mayor firmeza a los 60 días después de la recolección.

\section{5.- Agradecimientos}

Esta investigación fue realizada en el marco del proyecto REUSAGUA del Plan Estratégico de la Región de Murcia 2014-2020 (IRIS 2020) y el Fondo Europeo de Desarrollo Regional (FEDER).

\section{6- Bibliografía}

Artés, F., Marín, J. G., Martínez, J. A., Tudela, J. A., \& García-Lidón, A. (1997). Desverdización de nuevas variedades de limón y pomelo. Phytoma, 90, 130-135.

Conesa, M., De la Rosa, J., Fernández-Trujillo, J., Domingo, R., \& Pérez-Pastor, A. (2018). Deficit irrigation in commercial mandarin trees: water relations, yield and quality responses at harvest and after cold storage. Spanish Journal of Agricultural Research, 16(3).

Kimball, D. (1991). Brix and Soluble Solids BT - Citrus Processing: Quality Control and Technology. In D. Kimball (Ed.), Citrus Processing (pp. 7-33). Springer Netherlands. https://doi.org/10.1007/978-94-011-3700-3_2

Kimball, D., \& Kimball, D. (1991). Acids in Citrus Juices. In Citrus Processing (pp. 34-54). Springer Netherlands. https://doi.org/10.1007/978-94-011-3700-3_3

León, F. M. (2003). Las gotas frías/DANAs. Ideas y conceptos básicos.

Manuel Buitrago. (2019, September 13). Gota fría: La DANA rompe un canal del Trasvase y crea un enorme agujero | La Verdad. LA VERDAD. https://www.laverdad.es/murcia/molina/dana-rompe-canal-20190912183706nt.html?ref=https\%3A\%2F\%2Fwww.google.com\%2F

Navarro, J. M., Botía, P., \& Pérez-Pérez, J. G. (2015). Influence of deficit irrigation timing on the fruit quality of grapefruit (Citrus paradisi Mac.). Food Chemistry, 175, 329-336. https://doi.org/10.1016/J.FOODCHEM.2014.11.152

P.B. / EFE. (2019, September 19). Una DANA "sin precedentes" y con poca probabilidad de que se repita en un año - La Opinión de Murcia. La Opinión de Murcia. https://www.laopiniondemurcia.es/comunidad/2019/09/20/dana-precedentesprobabilidad-repita-ano/1053681.html

Pérez-Pérez, J. G., Robles, J. M., \& Botía, P. (2009). Influence of deficit irrigation in phase III of fruit growth on fruit quality in "lane late" sweet orange. Agricultural Water Management, 96(6), 969-974.

SIAM. (2019). SIAM - Sistema de Información Agraria de Murcia. Estación Molina de Segura (Campotejar). http://siam.imida.es/apex/f?p=101:1:2515579772808749::NO::: 